EPJ Web of Conferences 41, 05040 (2013)

DOI: $10.1051 /$ epjconf/20134105040

(C) Owned by the authors, published by EDP Sciences, 2013

\title{
Dissecting X-Ray Raman Resonances Using Four-Wave Mixing
}

\author{
Jason D. Biggs ${ }^{1}$, Yu Zhang ${ }^{1}$, Daniel Healion ${ }^{1}$, Niranjan Govind ${ }^{2}$, and Shaul Mukamel ${ }^{1, a}$ \\ 1 Department of Chemistry, University of California, Irvine, Irvine, CA 92697 \\ 2 William R. Wiley Environmental Molecular Sciences Laboratory, Pacific Northwest National Lab- \\ oratory, P. O. Box 999, Richland, Washington 99352, USA
}

\begin{abstract}
The stimulated x-ray Raman signal has been calculated for the amino acid cysteine using broadband ( $F W H M \simeq 14.2 \mathrm{eV}, 128$ as) pulses tuned to the nitrogen K-edge. Peaks correspond to those valence excited states and reveal electronic Frank-Condon overlaps between canonical valence orbitals and relaxed orbitals in the presence of the core hole. The coupling between excited states with valence- and core-holes is further explored using a coherent, wave-vector matched photon echo technique, where it is possible to eliminate stimulated emission and excited-state absorption by taking the waiting time to be longer the lifetime of the core hole ( $\sim 7.1$ fs for nitrogen).
\end{abstract}

\section{Introduction}

The one dimensional Stimulated X-ray Raman spectroscopy technique (1D-SXRS) may be used for probing valence electronic excitations in molecules via their coupling to high-energy core-electron excitations. This technique is made possible by new intense $\mathrm{x}$-ray pulsed light sources[1] Nonlinear $\mathrm{X}$-ray spectroscopy is an active area of research, with many people seeking to make use of these new sources.[2] Their intensity will allow the amplitude of an X-ray Raman process to be significant despite the low cross-section and short lifetimes of core-excitations. These all-x-ray simulations complement earlier studies on optical and IR pumped/x-ray probe experiments.[3]

\section{Ultrafast One Dimensional Stimulated Raman: 1D-SXRS}

1D-SXRS is the simplest time domain x-ray Raman technique. The system is subjected to two pulses with a delay $\tau$ and the transmission of the second pulse (the probe) is recorded $[4,5]$. Each pulse interacts with the system through a second-order stimulated Raman process. During each pulse, a core-electron is excited into an unoccupied orbital, the excited system evolves for a short period, and a second interaction with the pulse is a stimulated emission of an electron, destroying the core-hole. After interaction with the first x-ray pulse the system is left in a coherent superposition of valence excited states which interfere with the amplitude of the Raman process with the second pulse, leading to a change in its transmitted intensity. The dependence of this interference on the delay between pulses leads to the 1D-SXRS, a direct measurement of valence electronic motion. Taking the delay time $\tau$ to be long compared with the lifetime for core-excited states, Raman resonances dominate the pump-probe signal.[4]

The 1D-SXRS signal can be written

$$
S_{S X R S}(\tau)=\Re\left[\left\langle\alpha_{2}(\tau) \alpha_{1}(0)\right\rangle-\left\langle\alpha_{1}^{\dagger}(0) \alpha_{2}(\tau)\right\rangle\right]
$$

\footnotetext{
a e-mail: mukamel@uci.edu
}

This is an Open Access article distributed under the terms of the Creative Commons Attribution License 2.0, which permits unrestricted use, distribution, and reproduction in any medium, provided the original work is properly cited. 

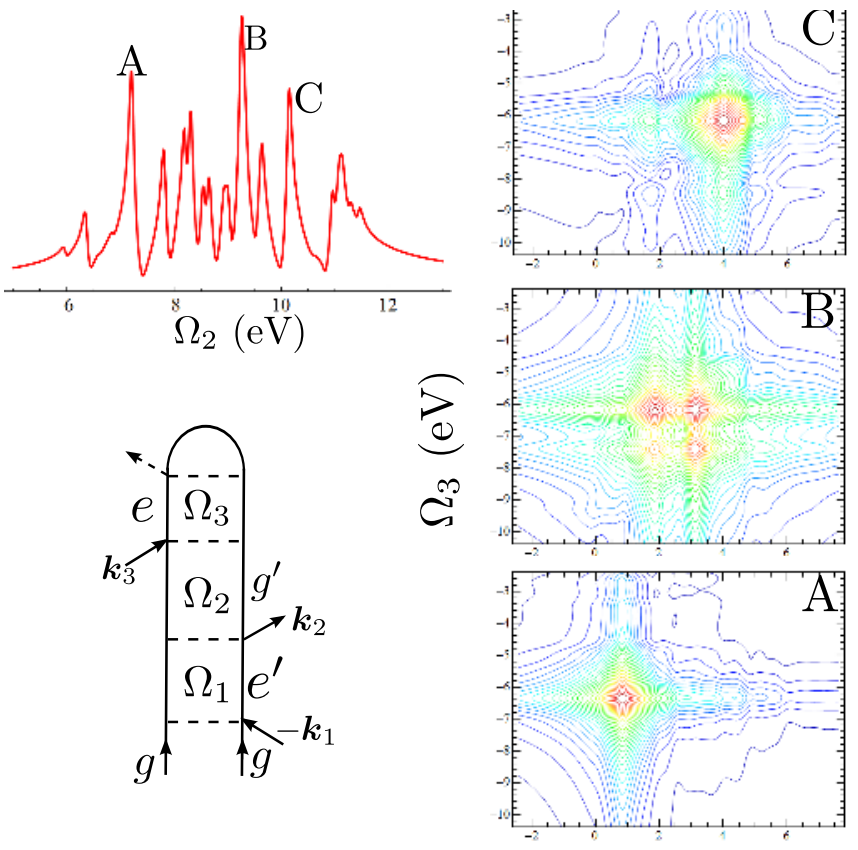

$\Omega_{1}(\mathrm{eV})$
Fig. 1. (Upper Left) 1D-SXRS signal from cysteine with pump and probe pulses tuned to the nitrogen K-edge. (Lower Left) Loop diagram representing both the 1D-SXRS process and the photon echo. Due to the short lifetime of coreexcited states, it is possible to retrieve only the ground-state bleach portion of the signal by keeping the waiting time time sufficiently long. The 1D-SXRS signal can also be obtained from the photon echo by summing over all values of $\Omega_{1}$ and $\Omega_{3}$. (Right Panel) $\Omega_{2}$ resolved photon echo spectra corresponding to the three largest peaks in the 1D-SXRS spectrum. Frequencies given are relative to the pulse center frequency, set to the nitrogen $\mathrm{K}$ edge at $401.7 \mathrm{eV}$.

where

$$
\alpha_{j ;}=\left|g^{\prime}\right\rangle \sum_{e} \frac{1}{2 \pi} V_{g^{\prime} e} \cdot V_{e g^{\prime \prime}} \int_{-\infty}^{\infty} d \omega_{2} \frac{\mathscr{E}_{j}^{*}\left(\omega_{2}\right) \mathscr{E}_{j}\left(\omega_{2}+\omega_{g^{\prime} g^{\prime \prime}}\right)}{\omega_{2}+\Delta_{e g^{\prime}}^{j}}\left\langle g^{\prime \prime}\right|
$$

is the effective polarizability weighted by the two-photon spectral density of the $j^{\text {th }}$ ultrashort pulse. In the upper left panel of Fig. 1 we show the modulus Fourier transform of the calculated 1D-SXRS from the amino acid cysteine. Valence- and core-excited states were found by TD-DFT at the CAMB3LYP/6-311G** level of theory, the latter using a restricted excitation window.[6] Peaks in the 1DSXRS spectrum show up at the valence-excitation frequencies of the system. Those excited states which are most perturbed by the presence of a core-hole show up most prominently.

\section{Three-Dimensional Photon Echo}

Raman techniques do not require any phase control of the pulses and may be performed in a simple collinear geometry. Since we use the core transitions as a trigger and only observe valence excitations, the time window is controlled by the lifetimes and dephasing times of valence states and can be hundreds of femtoseconds. The direct observation of core excitations is more tricky due to the shorter time window limited by Auger processes and the stringent requirements for phase control.

The $\mathbf{k}_{I}$ photon echo signal may be used to trace the origin of the Raman resonances that contribute to the 1D- and 2D-SXRS signal. We take $t_{2}$ to be long compared with the core-excited lifetimes allowing us to focus only on the GSB contribution, in the same way as discussed above with 1DSXRS. The signal is collected as a function of the three delay times $t_{1}, t_{2}$, and $t_{3}$, and subsequently Fourier transformed to give the 3D signal

$$
S_{\mathbf{k}_{1}}^{\mathrm{GSB}}\left(-\Omega_{1},-\Omega_{2}, \Omega_{3}\right)=\sum_{e_{1}, e_{2}, g} \frac{\mathscr{E}_{1}^{*}\left(\omega_{1}-\omega_{e^{\prime} g}\right) \mathscr{E}_{2}\left(\omega_{e^{\prime} g^{\prime}}-\omega_{2}\right) \mathscr{E}_{4}^{*}\left(\omega_{4}-\omega_{e g^{\prime}}\right) \mathscr{E}_{3}\left(\omega_{e g}-\omega_{3}\right) V_{g e} V_{e g^{\prime}} V_{g^{\prime} e^{\prime}} V_{e^{\prime} g}}{\left(-\Omega_{1}+\omega_{e^{\prime} g}+i \Gamma_{e^{\prime} g}\right)\left(-\Omega_{2}+\omega_{g^{\prime} g}+i \Gamma_{g^{\prime} g}\right)\left(\Omega_{3}-\omega_{e g^{\prime}}+i \Gamma_{e g^{\prime}}\right)}
$$


3D-Photon Echo

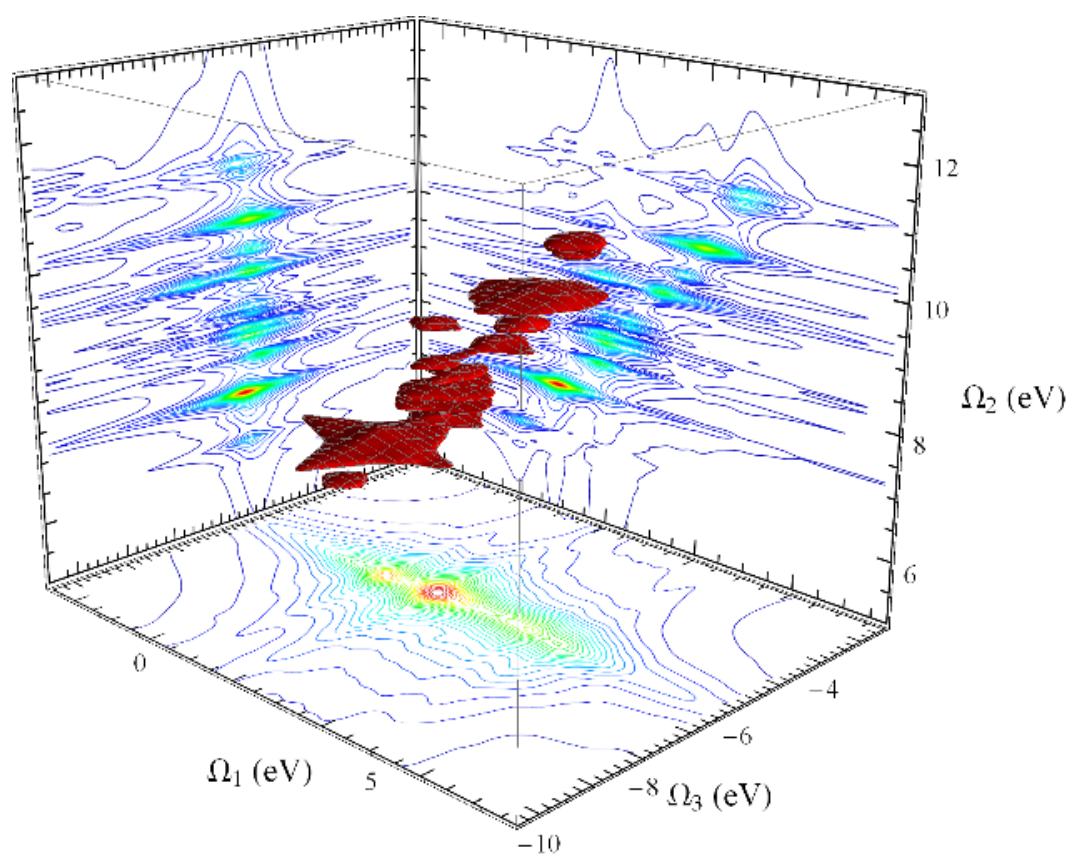

Fig. 2. (Upper Left) A 3D plot of $S_{\mathbf{k}_{1}}^{\mathrm{GSB}}\left(-\Omega_{1},-\Omega_{2}, \Omega_{3}\right)$ calculated for the amino acid cysteine, with all four Gaussian pulses tuned to the nitrogen core edge (FWHM 128 as, $14.2 \mathrm{eV}$ ), along with the $2 \mathrm{D}$ projections. We show only the $\Omega_{2}>0$ region to focus on the relation to the stimulated Raman signal, shown on the far left.

This expression reveals how the the pulse bandwidths select the various core transitions.

The signal is plotted in Fig. 2 for the amino acid cysteine, with all four Gaussian pulses again tuned to the nitrogen core edge (FWHM 128 as, $14.2 \mathrm{eV}$ ).[6] We focus on the $5 \mathrm{eV} \leq \Omega_{2} \geq 13 \mathrm{eV}$ region corresponding to the valence excited states which contribute to the 1D-SXRS spectrum. On the right side of Fig. 1 we also show horizontal 2D slices of the 3D plot for constant $\Omega_{2}$ values corresponding to the three most prominent peaks in the 1D-SXRS spectrum. In this way we are able to determine which core-excited state is coupled to which valence-excited state. For some of the valence states, e.g. peak A, there is only one core-excited state. This is indicative of a single-particle transition in which the core electron is promoted to an unoccupied valence orbital, following which an electron from an occupied valence orbital then falls in to fill the core hole, leaving a single electron-hole pair in the valence band. Two different core-excited states contribute to peak $\mathrm{B}$, indicating that this valence excitation may not be described using a single particle picture. For peak $\mathrm{C}$ there are multiple, nearly degenerate core-excited states that contribute. Thus the 3D photon echo can unravel the various pathways that contribute to a given SXRS peak.

\section{References}

1. J. Marangos, Contemp. Phys. 52, 551 (2011).

2. R. Santra, J. Phys. B At. Mol. Opt., 42(2), 023001 (2009).

3. V. C. Felici'ssimo, F. F. Guimara es, F. Gelmukhanov, A. Cesar, and H. ${ }^{\circ}$ gren, J. Chem. Phys., 122, 094319 (2005).

4. I. V. Schweigert and S. Mukamel, Phys. Rev. A, 76, 012504 (2007).

5. J. D. Biggs, Y. Zhang, D. Healion, and S. Mukamel, J. Chem. Phys. 136(17), 174117 (2012).

6. Y. Zhang, J. D. Biggs, D. Healion, N. Govind, and S. Mukamel, Under Preparation.

7. This work is supported by the Chemical Sciences, Geosciences and Biosciences Division, Office of Basic Energy Sciences, Office of Science, U.S. Department of Energy, the National Science Foundation (Grant CHE-1058791), and the National Institutes of Health (Grant GM-59230). N. G. thanks NWCHEM development (EMSL, PNNL) for support. 